\title{
An overview of the Normal Ogive Harmonic Analysis Robust Method (NOHARM) approach to item response theory
}

\author{
James J. Lee ${ }^{\mathrm{a}, 囚}$ and Minji K. Lee ${ }^{\mathrm{b}}$ \\ ${ }^{\mathrm{a}}$ University of Minnesota Twin Cities \\ ${ }^{\mathrm{b}}$ Mayo Clinic
}

Abstract — Here we provide a description of the IRT estimation method known as Normal Ogive Harmonic Analysis Robust Method (NOHARM). Although in some ways this method has been superseded by new computer programs that also adopt a specifically factor-analytic approach, its fundamental principles remain useful in certain applications, which include calculating the residual covariance matrix and rescaling the distribution of the common factor (latent trait). These principles can be applied to parameter estimates obtained by any method.

Keywords a factor analysis; item response theory; psychometrics.

leex2293@umn.edu

\section{Introduction}

Here we provide a description of the item response theory (IRT) estimation method known as Normal Ogive Harmonic Analysis Robust Method (NOHARM), which was implemented in a pioneering computer program developed by Fraser and McDonald (1988).

For some time NOHARM was the tool of choice for fitting multidimensional IRT models. To our knowledge, no other program until recently permitted the specification of a confirmatory multidimensional model with correlated factors or provided the residual covariance matrix in its output. Programs released within just the last few yearsincluding IRTPRO (Cai, Du Toit, \& Thissen, 2011) and the mirt package for the $\mathrm{R}$ computing platform (Chalmers, 2012) - now supply at least the first of these two desiderata, but they tend to be more computationally burdensome than NOHARM. Therefore it is important to weigh the relative advantages of the tools that are now available.

First, NOHARM does not allow the pseudo-guessing parameter-the probability of passing an item by a very low-ability examinee-to be estimated. In practice, a poor choice of a fixed value can lead to what factor analysts call a Heywood case (an estimated nonpositive residual variance). In most applications this theoretical problem is of little consequence, but it can be somewhat troublesome in analyses of differential item functioning. Second, whereas NOHARM only uses factor-analytic limited information (item pass rates and covariances) to estimate IRT parameters, many other programs use full information (the frequencies with which the possible zero-one strings of item responses occur in the data) (Bolt, 2005). In principle, this raises the possibility that NOHARM's estimates are statistically inefficient. NOHARM has performed well, however, in simulation studies of cases where it can be compared with other approaches. Moreover, there are useful objects in certain empirical analyses that continue to be most easily calculated using the principles of NOHARM, even if the parameter estimates have been obtained by some other method. In summary, whereas the latest generation of IRT programs is to be recommended, NOHARM still appears to have a place in the analysis of datasets containing hundreds of items (e.g., Flanagan et al., 1962). Such analyses promise to contribute much to differential psychology, and we anticipate that a greater recognition of the link between factor analysis and IRT will motivate such research.

Many critical pieces of information regarding NOHARM are not easy to obtain, as they are spread across articles, book chapters, and manuals published over a span of decades (McDonald, 1967, 1982, 1985, 1997, 1999; MaydeuOlivares, 2001; McDonald \& Fraser, 2003). Because the elegant principles of NOHARM continue to be enlightening and useful, we will try to produce a largely self-contained account of these principles that corrects the typographical errors that have unfortunately accrued in the relevant literature. 


\section{Function Approximation with Orthonormal Polynomials}

The basic principle of NOHARM is the approximation of the item characteristic curve (ICC) - the function giving the probability of the correct response as a function of the common factor(s) $\boldsymbol{\theta}$-with a polynomial that is convenient for computational purposes. It turns out that a linear combination of orthogonal polynomials serves this purpose; for an elementary account of such polynomials, including regularity conditions that we omit, the reader may consult the short monograph by Jackson (1941).

The sequence $\left(P_{k}\right)_{k=0}^{\infty}$ of $k$ th-degree orthonormal polynomials, with respect to the interval $(a, b)$ and the density function $g(x)$, is defined by

$$
\int_{a}^{b} P_{m}(x) P_{n}(x) g(x) d x=\delta_{m, n},
$$

where $\delta_{m, n}$ is the Kronecker delta.

The polynomials $P_{k}(x)$ can be used for the formal expansion of an arbitrary function

$$
f(x)=\sum_{k=0}^{\infty} c_{k} P_{k}(x)
$$

Multiplication of the expansion by $g(x) P_{k}(x)$ and integration from $a$ to $b$ gives

$$
c_{k}=\int_{a}^{b} f(x) P_{k}(x) g(x) d x,
$$

and in this way each coefficient can be obtained.

We now show that the best polynomial approximation of an arbitrary function ("best" in the sense of least squares) is the one given by Equation (2). Define the partial sum $s_{n}(x)$ as the termination of (2) after $n$ terms, $\sum_{k=0}^{n} c_{k} P_{k}(x)$. Also define the remainder $r_{n}(x)=f(x)-s_{n}(x)$. As a consequence of the definitions,

$$
\begin{aligned}
& \int_{a}^{b} s_{n}(x) P_{k}(x) g(x) d x=c_{k}, \\
& \int_{a}^{b} r_{n}(x) P_{k}(x) g(x) d x=0, \quad k=0,1, \ldots, n .
\end{aligned}
$$

Let $\pi_{n}(x)$ be an arbitrary polynomial of the $n$th degree at most. Then define

$$
\pi_{n}(x)-s_{n}(x)=\delta_{n}(x)=\sum_{k=0}^{n} d_{k} P_{k}(x),
$$

so that

$$
f(x)-\pi_{n}(x)=r_{n}(x)-\delta_{n}(x) .
$$

The integral of the squared error in $\pi_{n}(x)$ as an approximation of $f(x)$, weighted from point to point by the factor $g(x)$, is

$$
\begin{aligned}
& \int_{a}^{b} g(x)\left[f(x)-\pi_{n}(x)\right]^{2} d x \\
& =\int_{a}^{b} g(x)\left[r_{n}(x)-\delta_{n}(x)\right]^{2} d x \\
& =\int_{a}^{b} g(x)\left[r_{n}(x)\right]^{2} d x-2 \int_{a}^{b} g(x) r_{n}(x) \delta_{n}(x) d x \\
& +\int_{a}^{b} g(x)\left[\delta_{n}(x)\right]^{2} d x .
\end{aligned}
$$

But by virtue of Equation (4) and the expression of $\delta_{n}(x)$ in term of the $P_{k}$,

$$
\int_{a}^{b} g(x) r_{n}(x) \delta_{n}(x) d x=0, \quad \int_{a}^{b} g(x)\left[\delta_{n}(x)\right]^{2} d x=\sum_{k=0}^{n} d_{k}^{2} .
$$

As a result,

$$
\int_{a}^{b} g(x)\left[f(x)-\pi_{n}(x)\right]^{2} d x \geq \int_{a}^{b} g(x)\left[r_{n}(x)\right]^{2} d x .
$$

Evidently, regardless of whether $s_{n}(x)$ ultimately converges to $f(x)$, no other polynomial of degree $n$ or lower produces a smaller weighted integral of squared errors. The relevant implication for our purposes is that the appropriate sequence of orthonormal polynomials constitutes the optimal basis for approximation of the ICC.

\section{The Hermite Polynomials}

The interval $(a, b)$ and density function $g(x)$ determine the polynomials that satisfy the orthonormality condition given by Equation (1). In the case where the interval is the real line and the density function is associated with the standard normal distribution, the polynomials are known as the Hermite polynomials, which can be defined as

$$
P_{k}(x)=\frac{(-1)^{k} \sqrt{2 \pi}}{\sqrt{k !}} \exp \left(x^{2} / 2\right) \frac{d^{k}}{d x^{k}} \phi(x),
$$

where $\phi(x)$ is the standard normal density. The first ten Hermite polynomials are

$$
\begin{aligned}
& P_{0}(x)=1, \\
& P_{1}(x)=x \\
& P_{2}(x)=\left(x^{2}-1\right) / \sqrt{2 !} \\
& P_{3}(x)=\left(x^{3}-3 x\right) / \sqrt{3 !} \\
& P_{4}(x)=\left(x^{4}-6 x^{2}+3\right) / \sqrt{4 !} \\
& P_{5}(x)=\left(x^{5}-10 x^{3}+15 x\right) / \sqrt{5 !} \\
& P_{6}(x)=\left(x^{6}-15 x^{4}+45 x^{2}-15\right) / \sqrt{6 !}, \\
& P_{7}(x)=\left(x^{7}-21 x^{5}+105 x^{3}-105 x\right) / \sqrt{7 !}, \\
& P_{8}(x)=\left(x^{8}-28 x^{6}+210 x^{4}-420 x^{2}+105\right) / \sqrt{8 !}, \\
& P_{9}(x)=\left(x^{9}-36 x^{7}+378 x^{5}-1260 x^{3}+945 x\right) / \sqrt{9 !} .
\end{aligned}
$$

The Quantitative Methods for Psychology 
Figure 1 - The densities of 1,000 random draws from the bivariate normal distribution with zero means, unit variances, and correlation equal to 0.70 were calculated with both the true density function and the ninth order of Mehler's expansion.

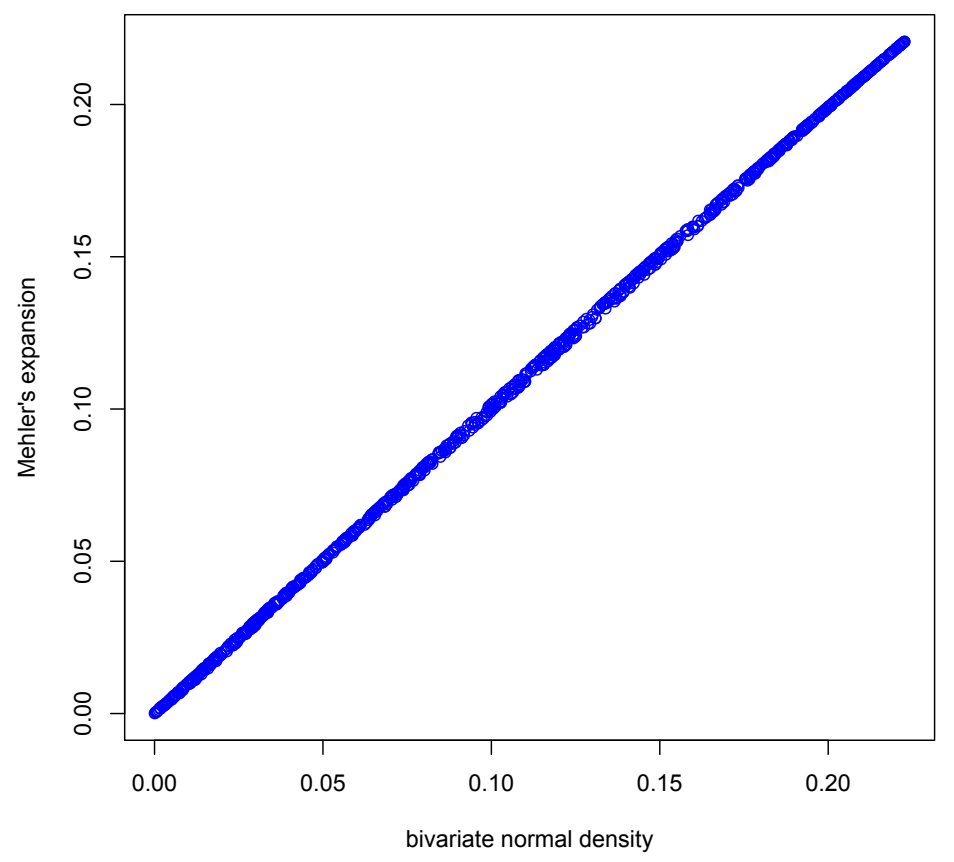

Other accounts may omit the normalizing constants $1 / \sqrt{k !}$ or give the polynomials in a slightly different form following from the non-normalized density function $\exp \left(-x^{2}\right)$.

Many texts provide an elementary proof that the Hermite polynomials satisfy the orthonormality property with respect to the standard normal density over the real line (e.g., Jackson, 1941). In any event a particular specific case is readily verified upon recognizing that upon integration Equation (1) becomes a linear combination of normal moments. Here we will set out a generalization of (1) that bears specifically on parameter estimation with NOHARM and the assessment of model fit: if $x$ and $y$ follow the bivariate normal distribution with zero means, unit variances, and correlation $\rho$, then

$$
\iint P_{m}(x) P_{n}(y) \phi(x, y) d x d y=\delta_{m, n} \rho^{m} .
$$

where $\phi(x, y)$ is the joint density. This result is a consequence of a remarkable identity known as Mehler's expansion,

$$
\phi(x, y)=\phi(x) \phi(y) \sum_{k=0}^{\infty} \rho^{k} P_{k}(x) P_{k}(y) .
$$

For small correlations Mehler's expansion is highly accurate even when truncated at a few terms. Figure 1 shows that even a density associated with a correlation as high as 0.70 can be approximated quite closely by taking the expansion to ninth order.

We close this section by proving Equation (6). Multiply both sides of Equation (7) by $P_{m}(x) P_{n}(y)$ to bring the left-hand side into conformity with the integrand of (6). Integrating both sides over $x$ eliminates all terms from the right-hand side except for

$$
P_{n}(y) \phi(y) \rho^{m} P_{m}(y),
$$

which when integrated over $y$ leads to (6).

\section{The Expansion of the Normal-Ogive Item Response Func-} tion

Recall that the normal-ogive ICC is given by

$$
\varphi_{j}(\theta)=\mathbb{E}\left(Y_{j} \mid \theta\right)=\int_{-\infty}^{\alpha_{j}+\beta_{j} \theta} \frac{1}{\sqrt{2 \pi}} \exp \left(-\frac{1}{2} t^{2}\right) d t .
$$

When the upper limit of the integrand is expressed in this way-with an intercept and slope parameter-we call Equation (8) the IRT parameterization of the ICC. The 
factor-analytic parameterization can be obtained by applying the transformation

$$
\begin{aligned}
& \tau_{j}=\frac{-\alpha_{j}}{\sqrt{1+\beta_{j}^{2}}}, \\
& \lambda_{j}=\frac{\beta_{j}}{\sqrt{1+\beta_{j}^{2}}},
\end{aligned}
$$

whereupon the upper limit of integration in Equation (8) becomes

$$
\frac{\lambda_{j} \theta-\tau_{j}}{\sqrt{1-\lambda_{j}^{2}}}
$$

The IRT parameterization derives its name from its similarity to the well-known formulation of Lord and Novick (1968). The factor-analytic parameterization, on the other hand, has the following interpretation. Suppose that the $j$ th item is associated with an underlying quantitative response tendency $Y_{j}^{*}$ and a threshold $\tau_{j}$ such that the observed item score

$$
y_{j}= \begin{cases}1 & \text { if } y_{j}^{*}>\tau_{j}, \\ 0 & \text { if } y_{j}^{*} \leq \tau_{j} .\end{cases}
$$

Now suppose that $\mathbf{y}^{*} \in \mathbb{R}^{p}$, the vector of response tendencies, fits the factor model

$$
\mathbf{y}^{*}=\boldsymbol{\Lambda} \boldsymbol{\theta}+\boldsymbol{\epsilon}^{*},
$$

where $\Lambda \in \mathbb{R}^{p \times q}$ is a matrix of factor loadings. Then the threshold and factor loading of item $j$ in this model are in fact given by Equation (9).

We wish to find the expansion of the normal-ogive ICC in terms of the Hermite polynomials. For this purpose it is convenient to use the transformation

$$
\begin{aligned}
\alpha_{j} & =\frac{-\mu_{j}}{\sigma_{j}}, \\
\beta_{j} & =\frac{1}{\sigma_{j}} .
\end{aligned}
$$

We now drop the item subscript $j$ until it is needed again.

\section{Case of $c_{0}$}

To obtain the first coefficient in the expansion, we must evaluate the integral

$$
\begin{aligned}
c_{0} & =\int \varphi(x) P_{0}(x) \phi(x) d x \\
& =\int_{-\infty}^{\infty} \frac{1}{\sqrt{2 \pi}} \int_{-\infty}^{(x-\mu) / \sigma} \exp \left(-\frac{z^{2}}{2}\right) \frac{1}{\sqrt{2 \pi}} \exp \left(-\frac{x^{2}}{2}\right) d z d x .
\end{aligned}
$$

Recall that the change-of-variables formula,

$$
\int_{g(\Omega)} f(\mathbf{x}) d \mathbf{x}=\int_{\Omega} f(g(\mathbf{u}))\left|\operatorname{det}(\mathrm{D} g)_{\mathbf{u}}\right| d \mathbf{u},
$$

often simplifies the evaluation of an integral. In this instance the appropriate change of variables is

$$
(u, v)=\left(\frac{\sigma x+z}{\sqrt{1+\sigma^{2}}}, \frac{-x+\sigma z}{\sqrt{1+\sigma^{2}}}\right),
$$

which has the inverse

$$
(x, z)=\left(\frac{\sigma u-v}{\sqrt{1+\sigma^{2}}}, \frac{u+\sigma v}{\sqrt{1+\sigma^{2}}}\right) .
$$

Note that the Jacobian matrix of this transformation,

$$
\left[\begin{array}{ll}
\frac{\partial u}{\partial x} & \frac{\partial u}{\partial z} \\
\frac{\partial v}{\partial x} & \frac{\partial v}{\partial z}
\end{array}\right]=\left[\begin{array}{cc}
\frac{\sigma}{\sqrt{1+\sigma^{2}}} & \frac{1}{\sqrt{1+\sigma^{2}}} \\
\frac{-1}{\sqrt{1+\sigma^{2}}} & \frac{\sigma}{\sqrt{1+\sigma^{2}}}
\end{array}\right]
$$

has a determinant equal to unity.

In our case, the transformation converts the line $z=$ $(x-\mu) / \sigma$ into the line $v=-\mu / \sqrt{1+\sigma^{2}}$, and we have

$$
\begin{aligned}
c_{0} & =\int_{u=-\infty}^{\infty} \frac{1}{\sqrt{2 \pi}} \int_{v=-\infty}^{-\mu / \sqrt{1+\sigma^{2}}} \exp \left[-\frac{1}{2} \frac{(u+\sigma v)^{2}}{1+\sigma^{2}}\right] \frac{1}{\sqrt{2 \pi}} \exp \left[-\frac{1}{2} \frac{(\sigma u-v)^{2}}{1+\sigma^{2}}\right] d u d v \\
& =\int_{u=-\infty}^{\infty} \int_{v=-\infty}^{-\mu / \sqrt{1+\sigma^{2}}} \frac{1}{\sqrt{2 \pi}} \exp \left(-\frac{u^{2}}{2}\right) d u \frac{1}{\sqrt{2 \pi}} \exp \left(-\frac{v^{2}}{2}\right) d v \\
& =\frac{1}{\sqrt{2 \pi}} \int_{v=-\infty}^{-\mu / \sqrt{1+\sigma^{2}}} \exp \left(-\frac{v^{2}}{2}\right) d v \\
& =\Phi\left(-\frac{\mu}{\sqrt{1+\sigma^{2}}}\right),
\end{aligned}
$$


where we now use $\Phi$ to denote the cumulative probability function of the standard normal distribution. Using Equations (9) and (12), we see that

$$
c_{0}=\Phi(-\tau)
$$

\section{Case of $c_{1}$}

Now we must evaluate the integral

$$
\begin{aligned}
c_{1} & =\int \varphi(x) P_{1}(x) \phi(x) d x \\
& =\int_{-\infty}^{\infty} \frac{1}{\sqrt{2 \pi}} \int_{-\infty}^{(x-\mu) / \sigma} \exp \left(-\frac{z^{2}}{2}\right) \frac{1}{\sqrt{2 \pi}} x \exp \left(-\frac{x^{2}}{2}\right) d z d x .
\end{aligned}
$$

Employing the same change of variables used to obtain $c_{0}$, we get

$$
\begin{aligned}
c_{1} & =\int_{u=-\infty}^{\infty} \int_{\nu=-\infty}^{-\mu / \sqrt{1+\sigma^{2}}} \frac{\sigma u-v}{\sqrt{1+\sigma^{2}}} \frac{1}{\sqrt{2 \pi}} \exp \left(-\frac{u^{2}}{2}\right) d u \frac{1}{\sqrt{2 \pi}} \exp \left(-\frac{v^{2}}{2}\right) d v \\
& =\int_{u=-\infty}^{\infty} \int_{\nu=-\infty}^{-\mu / \sqrt{1+\sigma^{2}}}-\frac{v}{\sqrt{1+\sigma^{2}}} \frac{1}{\sqrt{2 \pi}} \exp \left(-\frac{u^{2}}{2}\right) d u \frac{1}{\sqrt{2 \pi}} \exp \left(-\frac{v^{2}}{2}\right) d v \\
& =\frac{1}{\sqrt{1+\sigma^{2}}} \int_{\nu=-\infty}^{-\mu / \sqrt{1+\sigma^{2}}}-\frac{1}{\sqrt{2 \pi}} v \exp \left(-\frac{v^{2}}{2}\right) d v
\end{aligned}
$$

Since the integrand has been reduced to the derivative of the standard normal density function, we can now write

$$
c_{1}=\frac{1}{\sqrt{1+\sigma^{2}}} \phi\left(-\frac{\mu}{\sqrt{1+\sigma^{2}}}\right)
$$

where $\phi$ represents the standard normal density function. By Equations (9) and (12), this can be rewritten as

$$
c_{1}=\lambda \phi(\tau) .
$$

\section{Case of $c_{2}$}

We have

$$
\begin{aligned}
c_{2} & =\int \varphi(x) P_{2}(x) \phi(x) d x \\
& =\int_{-\infty}^{\infty} \frac{1}{\sqrt{2 \pi}} \int_{-\infty}^{(x-\mu) / \sigma} \exp \left(-\frac{z^{2}}{2}\right) \frac{1}{\sqrt{2 \pi}} \frac{x^{2}-1}{\sqrt{2}} \exp \left(-\frac{x^{2}}{2}\right) d z d x .
\end{aligned}
$$

The same change of variables yields

$$
\begin{aligned}
c_{2} & =\frac{1}{\sqrt{2}\left(1+\sigma^{2}\right)} \\
& \times \int_{u=-\infty}^{\infty} \int_{\nu=-\infty}^{-\mu / \sqrt{1+\sigma^{2}}}\left[(\sigma u-v)^{2}-1-\sigma^{2}\right] \phi(u) d u \phi(v) d v .
\end{aligned}
$$

With the help of Mathematica, we can evaluate the integral to get

$$
c_{2}=\frac{1}{\sqrt{2}\left(1+\sigma^{2}\right)} \frac{\mu}{\sqrt{1+\sigma^{2}}} \phi\left(-\frac{\mu}{\sqrt{1+\sigma^{2}}}\right)=\frac{1}{\sqrt{2}} \lambda^{2} \tau \phi(\tau) .
$$

\section{Case of $c_{3}$}

Because the fit to the sample covariance matrix often cannot be improved more than negligibly by taking the expansion of each ICC beyond the third power, this is the last coefficient used by the NOHARM program to compute its parameter estimates. We have

$$
\begin{aligned}
c_{3} & =\int \varphi(x) P_{2}(x) \phi(x) d x \\
& =\int_{-\infty}^{\infty} \frac{1}{\sqrt{2 \pi}} \int_{-\infty}^{(x-\mu) / \sigma} \exp \left(-\frac{z^{2}}{2}\right) \frac{1}{\sqrt{2 \pi}} \frac{x^{3}-3 x}{\sqrt{6}} \exp \left(-\frac{x^{2}}{2}\right) d z d x .
\end{aligned}
$$

With our change of variables and the help of Mathematica, we get

$$
\begin{aligned}
c_{3} & =\frac{1}{\sqrt{6}} \frac{1}{\left(1+\sigma^{2}\right)^{3 / 2}}\left(\frac{\mu^{2}}{1+\sigma^{2}}-1\right) \phi\left(-\frac{\mu}{\sqrt{1+\sigma^{2}}}\right) \\
& =\frac{1}{\sqrt{3}} \lambda^{3} \frac{\tau^{2}-1}{\sqrt{2}} \phi(\tau) .
\end{aligned}
$$

\section{The General Expression for $c_{k}, k \geq 1$}

Upon studying Equations (14), (15), and (16), we might conjecture that the general expression is

$$
c_{k}=\frac{1}{\sqrt{k}} \lambda^{k} P_{k-1}(\tau) \phi(\tau), \quad k \geq 1 .
$$

McDonald (1967) gave a proof of Equation (17) using Fourier transforms.

The Quantitative Methods for P sychology 
Figure 2 - Increasingly higher-order expansions in Hermite polynomials of a normal-ogive item characteristic curve corresponding to $\tau=-0.4, \lambda=0.8$, and $c=0.2$.

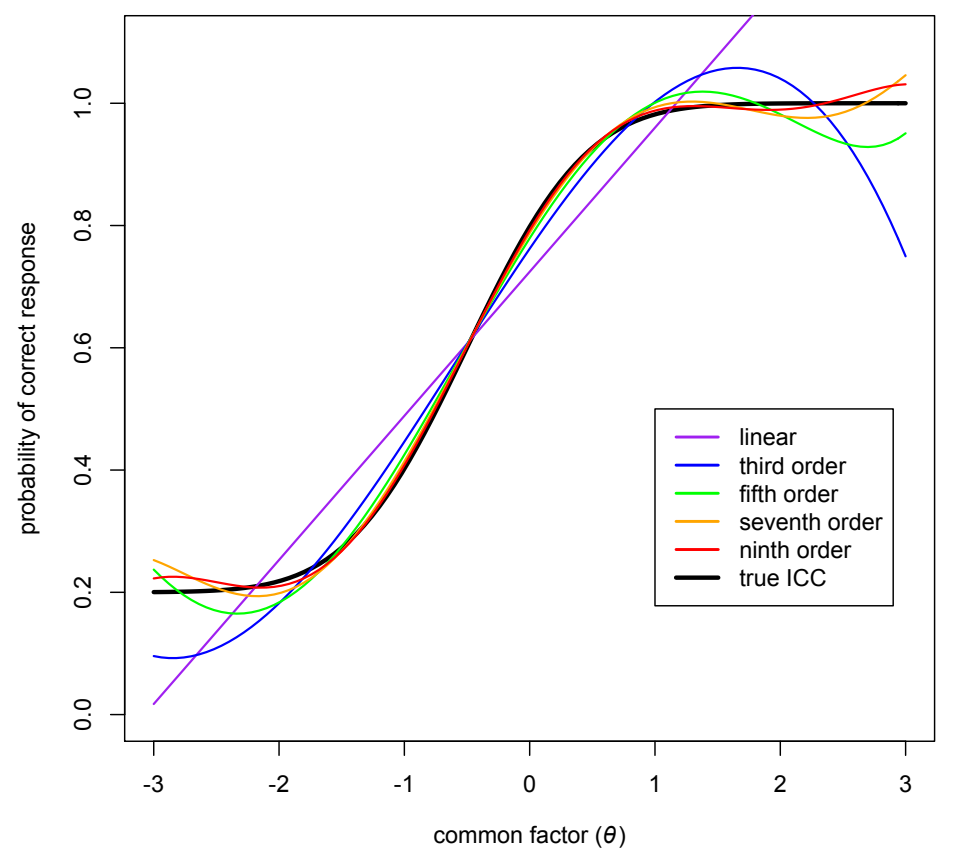

Figure 2 shows the quality of the ICC approximation for odd values of $k$ up to 9 . As one should expect in the case of a easy item, the linear approximation fails at high levels of $\theta$. The NOHARM program takes the expansion in Hermite polynomials to third order, which still does not closely match the ICC at the extremes of the $\theta$ distribution. Nevertheless third order seems sufficient for accurate parameter estimation in the case of a pseudo-guessing parameter equal to zero.

\section{Generalization to Multiple Dimensions}

We now treat multiple common factors (latent traits) collected in the vector $\boldsymbol{\theta} \in \mathbb{R}^{q}$. In the IRT parameterization of the ICC, the discrimination parameters of a given item are collected in the vector $\boldsymbol{\beta} \in \mathbb{R}^{q}$. It is convenient to find a transformation of $\boldsymbol{\theta}$ such that the multidimensional equivalent of Equation (8) - where the upper limit of integration becomes $\alpha+\boldsymbol{\beta}^{\prime} \boldsymbol{\theta}$-is in fact a function of only one element (say the first). Define

$$
\mathbf{T}=\frac{1}{\delta}\left[\begin{array}{c}
\boldsymbol{\beta}^{\prime} \\
\boldsymbol{\beta}_{2}^{\prime} \\
\vdots \\
\boldsymbol{\beta}_{q}^{\prime}
\end{array}\right]
$$

where

$$
\delta=\boldsymbol{\beta}^{\prime} \boldsymbol{\beta}
$$

and the rows of $\mathbf{T}$ after the first are chosen so that the matrix is orthonormal. Now define

$$
\boldsymbol{\theta}^{*}=\frac{\delta}{d} \mathbf{T} \boldsymbol{\theta},
$$

where

$$
d^{2}=\boldsymbol{\beta}^{\prime} \Psi \boldsymbol{\beta}, \quad \boldsymbol{\Psi}=\operatorname{Cov}(\boldsymbol{\theta}) .
$$

In particular, the first element of $\boldsymbol{\theta}^{*}$ is

$$
\theta^{*}=\frac{1}{d} \boldsymbol{\beta}^{\prime} \boldsymbol{\theta}
$$

The inverse of Equation (18) is

$$
\boldsymbol{\theta}=\frac{d}{\delta} \mathbf{T}^{\prime} \boldsymbol{\theta}^{*},
$$


which implies that

$$
\Phi\left(\alpha+\boldsymbol{\beta}^{\prime} \boldsymbol{\theta}\right)=\Phi\left(\alpha+\frac{d}{\delta} \boldsymbol{\beta}^{\prime} \mathbf{T}^{\prime} \boldsymbol{\theta}^{*}\right) .
$$

Since, by construction,

$$
\boldsymbol{\beta}^{\prime} \mathbf{T}^{\prime}=\left[\begin{array}{llll}
\delta & 0 & \cdots & 0
\end{array}\right],
$$

we thus have

$$
\Phi\left(\alpha+\boldsymbol{\beta}^{\prime} \boldsymbol{\theta}\right)=\Phi\left(\alpha+d \times \theta^{*}\right) .
$$

This is a function of the single variable $\theta^{*}$ and thus has the same form as Equation (8). We can therefore approximate the ICC with

$$
\varphi(\boldsymbol{\theta}) \approx c_{0}+\sum_{k=1} c_{k} P_{k}\left(\frac{\boldsymbol{\beta}^{\prime} \boldsymbol{\theta}}{\sqrt{\boldsymbol{\beta}^{\prime} \boldsymbol{\Psi} \boldsymbol{\beta}}}\right),
$$

replacing $\beta_{1}$ in our previous expressions with $d$. Consulting Equation (9), we see that the replacement leads to

$$
\begin{aligned}
& c_{0}=\Psi\left(\frac{\alpha}{\sqrt{1+\boldsymbol{\beta}^{\prime} \Psi \boldsymbol{\beta}}}\right), \\
& c_{1}=\sqrt{\frac{\boldsymbol{\beta}^{\prime} \Psi \boldsymbol{\beta}}{1+\boldsymbol{\beta}^{\prime} \Psi \boldsymbol{\beta}}} \times \phi\left(\frac{\alpha}{\sqrt{1+\boldsymbol{\beta}^{\prime} \Psi \boldsymbol{\beta}}}\right),
\end{aligned}
$$

and so forth.

\section{The Expected Covariance Between Two Items}

We can write the response to item $j$ as

$$
Y_{j}=c_{j}+\left(1-c_{j}\right)\left[c_{j 0} P_{0}\left(\theta_{j}^{*}\right)+c_{j 1} P_{1}\left(\theta_{j}^{*}\right)+c_{j 2} P_{2}\left(\theta_{j}^{*}\right)+\cdots\right]
$$

where we have introduced the pseudo-guessing parameter with the symbol $c_{j}$.

The response can then be rewritten as

$$
Y_{j}=b_{j 0} P_{0}\left(\theta_{j}^{*}\right)+b_{j 1} P_{1}\left(\theta_{j}^{*}\right)+b_{j 2} P_{2}\left(\theta_{j}^{*}\right)+\cdots,
$$

where

$$
\begin{aligned}
& b_{j 0}=c_{j}+\left(1-c_{j}\right) c_{j 0}, \\
& b_{j 1}=\left(1-c_{j}\right) c_{j 1}, \\
& b_{j 2}=\left(1-c_{j}\right) c_{j 2},
\end{aligned}
$$

and so on.

We collect the linear combinations $\theta_{j}^{*}$ into a single vector $\boldsymbol{\theta}^{\star} \in \mathbb{R}^{p}$ and assume that the transformation $\boldsymbol{\theta} \rightarrow \boldsymbol{\theta}^{\star}$ is one-to-one. Now consider the two items $j$ and $j^{\prime}$. We have

$$
\begin{aligned}
\mathbb{E}\left(Y_{j} Y_{j^{\prime}}\right)= & \mathbb{E}_{\boldsymbol{\theta}^{\star}}\left[\left[\mathbb{E}\left(Y_{j} Y_{j^{\prime}} \mid \boldsymbol{\theta}^{\star}\right)\right]\right. \\
= & \mathbb{E}_{\boldsymbol{\theta}^{\star}}\left[\mathbb{E}\left(Y_{j} \mid \boldsymbol{\theta}^{\star}\right) \mathbb{E}\left(Y_{j^{\prime}} \mid \boldsymbol{\theta}^{\star}\right)\right] \\
= & \mathbb{E}_{\boldsymbol{\theta}^{\star}}\left\{\left[b_{j 0} P_{0}\left(\theta_{j}^{*}\right)+b_{j 1} P_{1}\left(\theta_{j}^{*}\right)+\cdots\right]\right. \\
& \left.\times\left[b_{j^{\prime} 0} P_{0}\left(\theta_{j^{\prime}}^{*}\right)+b_{j^{\prime} 1} P_{1}\left(\theta_{j^{\prime}}^{*}\right)+\cdots\right]\right\} .
\end{aligned}
$$

The argument in brackets resolves into terms of two types: products of the form

$$
b_{j k} b_{j^{\prime} k} P_{k}\left(\theta_{j}^{*}\right) P_{k}\left(\theta_{j^{\prime}}^{*}\right)
$$

and cross-products where the indices of the Hermite polynomials do not agree. The multivariate normality of $\boldsymbol{\theta}$ is a sufficient condition for the use of Equation (6), which upon integration leads the cross-products to vanish and the products to become

$$
b_{j k} b_{j^{\prime} k}\left[\operatorname{Corr}\left(\theta_{j}^{*}, \theta_{j^{\prime}}^{*}\right)\right]^{k} \text {, }
$$

and since the variance of each $\theta_{j}^{*}$ remains unity if all elements of $\boldsymbol{\theta}$ are standardized, we then have

$$
\mathbb{E}\left(Y_{j} Y_{j^{\prime}}\right)=\sum_{k=0} b_{j k} b_{j^{\prime} k}\left(\frac{\boldsymbol{\beta}_{j}^{\prime} \boldsymbol{\Psi} \boldsymbol{\beta}_{j^{\prime}}}{d_{j} d_{j^{\prime}}}\right)^{k},
$$

from which the covariance is obtained by subtracting the product of the marginal pass rates. With estimates of the item parameters in hand, however obtained, one can use Equation (20) to compute the residual covariance matrix as part of assessing model-data fit.

Equation (20) is in fact used by NOHARM to obtain estimates of the item parameters. Each $\tau_{j}$ is estimated in close form by equating item $j$ 's pass rate to $\Phi(-\tau)$, and the item parameters can in principle be estimated by choosing the $\widehat{\boldsymbol{\beta}}_{j}$ and $\widehat{c}_{j}$ to minimize

$$
\sum_{j=1}^{p} \sum_{j>j^{\prime}}\left[\overline{y_{j} y_{j^{\prime}}}-\mathbb{E}_{n}\left(Y_{j} Y_{j^{\prime}}\right)\right]^{2},
$$

where $\mathbb{E}_{n}\left(Y_{j} Y_{j^{\prime}}\right)$ is the truncation of (20) at $n$th order. In the current implementation of NOHARM, $n$ is set equal to 3. The $c_{j}$ must be fixed by the user because the triplets $\left(\alpha_{j}, \boldsymbol{\beta}_{j}, c_{j}\right)$ are nearly unidentified when this approach is employed, but Figure 2 suggests that taking the expansion to higher order might overcome this difficulty.

\section{Authors' note}

Correspondence concerning this article should be addressed to James J. Lee, Department of Psychology, University of Minnesota Twin Cities, Minneapolis, MN 55455. Email: leex2293@umn.edu 


\section{References}

Bolt, D. M. (2005). Limited- and full-information estimation of item response models. In A. Maydeu-Olivares \& J. J. McArdle (Eds.), Contemporary psychometrics: A festschrift for Roderick P. McDonald (pp. 27-71). Mahwah, NJ: Erlbaum.

Cai, L., Du Toit, S. H. C., \& Thissen, D. (2011). IRTPRO: Flexible, multidimensional, multiple categorial IRT modeling. Chicago, IL: Scientific Software International.

Chalmers, R. P. (2012). mirt: A multidimensional item reponse theory package for the $\mathrm{R}$ environment. Journal of Statistical Software, 48, 1-29.

Flanagan, J. C., Dailey, J. T., Shaycroft, M. F., Gorham, W. A., Orr, D. B., \& Goldberg, I. (1962). Design for a study of American youth. Boston, MA: Houghton Mifflin.

Fraser, C. \& McDonald, R. P. (1988). NOHARM: Least squares item factor analysis. Multivariate $\mathrm{Be}$ havioral Research, 23, 267-269. doi:10 . 1207 / s15327906mbr2302_9

Jackson, D. (1941). Fourier series and orthogonal polynomials. Oberlin, OH: Mathematical Association of America.

Lord, F. M. \& Novick, M. R. (1968). Statistical theories of mental test scores. Reading, MA: Addison-Wesley.

Maydeu-Olivares, A. (2001). Multidimensional item response theory modeling of binary data: Large sample properties of NOHARM estimates. Journal of Ed- ucational and Behavioral Statistics, 26, 51-71. doi:10. 3102/10769986026001051

McDonald, R. P. (1967). Nonlinear factor analysis. Richmond, VA: Psychometric Corporation.

McDonald, R. P. (1982). Some alternative approaches to the improvement of measurement in education and psychology: Latent trait models. In D. Spearritt (Ed.), The improvement of measurement in education and psychology (pp. 213-237). Hawthorn, Australia: Australian Council for Educational Research.

McDonald, R. P. (1985). Unidimensional and multidimensional models for item response theory. In D. J. Weiss (Ed.), Proceedings of the 1982 Item Response Theory and Computerized Adaptive Testing Conference (pp. 127-148). Minneapolis, MN: University of Minnesota Press.

McDonald, R. P. (1997). Normal-ogive multidimensional model. In W. J. van der Linden \& R. K. Hambleton (Eds.), Handbook of modern item response theory (pp. 257-269). New York, NY: Springer. doi:10.1007 / 978-1-4757-2691-6_14

McDonald, R. P. (1999). Test theory: A unified treatment. Mahwah, NJ: Erlbaum.

McDonald, R. P. \& Fraser, C. (2003). NOHARM: A Windows program for fitting both unidimensional and multidimensional normal ogive models of latent trait theory. Niagara College. Welland, Ontario. Retrieved from http://noharm.niagararesearch.ca/nh4man/nhman. html

\section{Citation}

Lee, J. J., Lee, M. K. (2016) An overview of the Normal Ogive Harmonic Analysis Robust Method (NOHARM) approach to item response theory. The Quantitative Methods for Psychology, 12(1), 1-8. 